\title{
Staphylococcus aureus resistentes à meticilina: disseminação emergente na comunidade *
}

\author{
Methicillin-resistant Staphylococcus aureus: emerging community dissemination
}

\author{
Luciane Cristina Gelatti ${ }^{1}$ \\ Ana Paula Becker ${ }^{3}$
}

\author{
Renan Rangel Bonamigo ${ }^{2}$ \\ Pedro Alves d'Azevedo ${ }^{4}$
}

\begin{abstract}
Resumo: Staphylococcus aureus é uma bactéria responsável por uma ampla variedade de enfermidades infecciosas. A grande preocupação está relacionada, principalmente, com os isolados resistentes à meticilina (MRSA), que, tradicionalmente, estavam limitados aos hospitais. Nos últimos anos, infecções causadas por MRSA associadas ou adquiridas na comunidade (CA-MRSA) têm sido relatadas com frequência crescente em todo o mundo. Algumas características fenotípicas e genéticas são distintas entre a forma de infecção hospitalar e a comunitária. Atualmente, verifica-se um perfil de sensibilidade reduzido para diferentes antimicrobianos; sendo assim faz-se necessário um alerta aos profissionais da saúde, particularmente aos dermatologistas, para a importância da distinção entre as formas de infecções, evitando uma terapia empírica incorreta e sem sucesso.
\end{abstract}

Palavras-chave: Infecções bacterianas; Meticilina; Staphylococcus aureus

\begin{abstract}
Staphylococcus aureus is responsible for a broad variety of infectious diseases. The main concern is about methicillin-resistant isolates (MRSA), which are usually limited to hospitals. In recent years, community associated or acquired MRSA infections (CA-MRSA) have been frequently reported and emerged in the world. Some phenotypic and genotypic characteristics are distinct between hospital and community infection. Currently, there is reduced sensibility profile to different antimicrobials, reason why it is necessary to issue an alert to healthcare professionals, dermatologists in particular, about the importance of knowing the differences between the infections, preventing wrong and unsuccessful empirical therapy.

Keywords: Bacterial Infections; Methicillin; Staphylococcus aureus
\end{abstract}

\section{INTRODUÇÃO}

Staphylococcus aureus é encontrado colonizando a flora natural, principalmente da pele, podendo tornar-se patogênico em condições como a quebra da barreira cutânea ou diminuição da imunidade. Os traumas que comprometem a integridade da barreira cutânea constituem-se na principal causa de mudança de comportamento desse microrganismo para agente etiológico mais comum de infecções cutâneas. ${ }^{1,2,3}$ É responsável por uma grande variedade de infecções, como infecções na pele e no subcutâneo, infecções pós-cirúrgicas, osteomielites, pneumonias, abscessos, endocardites e bacteremia. ${ }^{4}$ É umas das causas mais comuns de infecções nosocomiais, bem como de infecções comunitárias que podem apresentar altos índices de morbidade e mortalidade.

\footnotetext{
Aprovado pelo Conselho Editorial e aceito para publicação em 10.02.09.

* Estudo realizado no programa de pós-graduação, Departamento de Microbiologia e Parasitologia da Universidade Federal de Ciências da Saúde de Porto Alegre (UFCSPA) - Porto Alegre (RS), e no Serviço de Dermatologia da UFCSPA - Porto Alegre (RS), Brasil.

Conflito de interesse: Nenhum. / Conflict of interest: None

Suporte financeiro: Nenhum. / Financial Funding: None

Especialista em citologia clínica, mestranda em patologia pela Universidade Federal de Ciências da Saúde de Porto Alegre (UFCSPA) - Porto Alegre (RS), Brasil Especialista em dermatologia pela Sociedade Brasileira de Dermatologia, doutor em ciências médicas pela Universidade Federal do Rio Grande do Sul (UFRGS), professor adjunto e chefe do Serviço de Dermatologia da Universidade Federal de Ciências da Saúde de Porto Alegre (UFCSPA) - Porto Alegre (RS) Brasil.

Biomédica, mestranda em ciências médicas pela Universidade Federal de Ciências da Saúde de Porto Alegre (UFCSPA) - Porto Alegre (RS), Brasil.

Doutor, professor adjunto do Departamento de Microbiologia e Parasitologia da Universidade Federal de Ciências da Saúde de Porto Alegre (UFCSPA) - Porto Alegre (RS), Brasil.
}

(C)2009 by Anais Brasileiros de Dermatologia 
A terapia antimicrobiana para infecções por esse microrganismo inicialmente era simples. A primeira vez que um antimicrobiano foi utilizado clinicamente foi contra uma amostra de $S$. aureus, a partir da descoberta da penicilina, que funcionou muito bem até a década de 1960, quando começaram a aparecer isolados resistentes a esse antimicrobiano. Para contornar o problema, foi criado o beta-lactâmico sintético meticilina, que era resistente à ação das beta-lactamases que o $S$. aureus produzia. Entretanto, logo após o advento da meticilina, surgiram relatos de amostras resistentes também a esse antimicrobiano, além da expressão de multirresistência. Essas cepas foram denominadas de MRSA (Staphylococcus aureus resistente à meticilina) e são resistentes a todos os antimicrobianos beta-lactâmicos. ${ }^{4}$

Os antimicrobianos beta-lactâmicos se ligam a proteínas que participam da síntese da parede celular, chamadas PBPs (proteínas ligadoras de penicilina), impedindo a formação da parede celular e resultando em lise bacteriana. $\mathrm{O}$ mecanismo de resistência à meticilina está relacionado ao desenvolvimento de uma PBP adicional, a PBP2a, que é plenamente funcional, mas não tem afinidade por antimicrobianos beta-lactâmicos. A codificação dessas novas PBPs, tornando esses patógenos resistentes à oxacilina, está relacionada à aquisição do gene mecA, o qual faz parte de um elemento genético móvel detectado em isolados de MRSA. Esse gene é parte integrante de um elemento genômico denominado "cassete cromossômico estafilocócico mec" (SCCmec). ${ }^{5}$

Tradicionalmente, as infecções causadas pelo MRSA estavam limitadas aos hospitais (HA-MRSA); mas, nos últimos anos, as infecções associadas ou adquiridas na comunidade (CA-MRSA) estão sendo documentadas de forma crescente em todo o mundo (Gráfico 1). ${ }^{6}$ Epidemiologicamente, isolados clínicos de MRSA são definidos como CA-MRSA se coletados de pacientes ambulatoriais, ou coletados até 48 horas após admissão hospitalar. Fatores de risco, como hospitalização recente, procedimentos cirúrgicos, uso de cateter venoso ou dispositivos intravasculares e cutâneos de longa permanência e internação em casa de repouso, devem ser excluídos.

\section{S. AUREUS RESISTENTES À METICILINA ADQUIRIDOS NA COMUNIDADE}

Além de representar um dos principais patógenos associados a infecções hospitalares, o $S$. aureus é também um dos principais agentes isolados de pacientes com infecções de pele e subcutâneo adquiridas na comunidade, incluindo foliculites, impetigos, celulites e erisipelas. ${ }^{8}$ Diferentemente do HA-MRSA, que carrega o elemento genético móvel denominado cassete cromossômico estafilocócico mec (SCCmec) dos tipos I, II

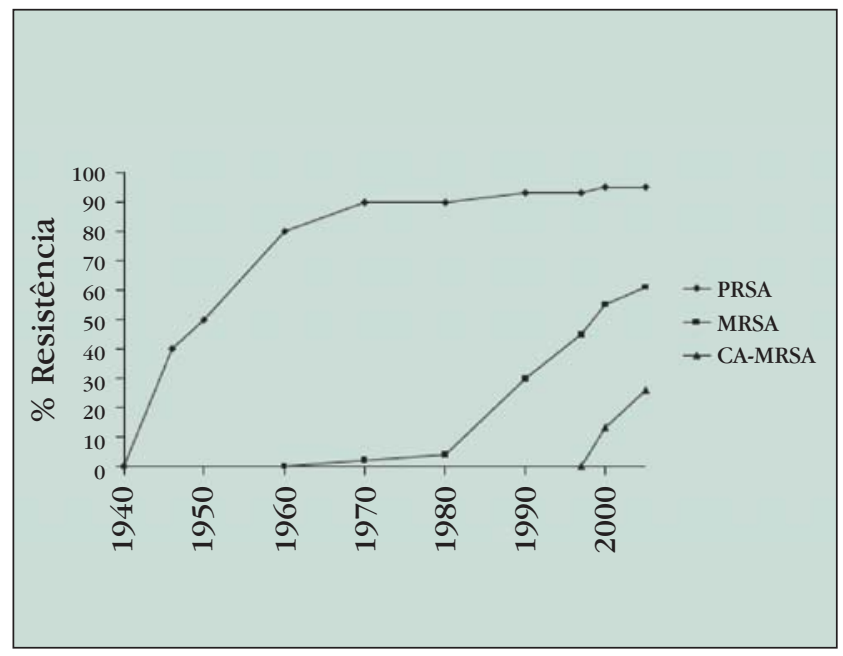

GráfICo 1: Evolução da resistência aos antimicrobianos em isolados de Staphylococcus aureus

PRSA: $S$. aureus resistentes à penicilina

MRSA: $S$. aureus resistentes à meticilina

CA-MRSA: $S$. aureus resistentes à meticilina adquiridos

ou associados à comunidade.

Fonte: Bustos-Martínez JA et al. ${ }^{6}$

e III, o CA-MRSA carrega preferencialmente o SCCmec do tipo IV e eventualmente o do tipo V. Esse tipo de cassete cromossômico é menor que os outros tipos e não possui genes acoplados que codificam resistência a outros antimicrobianos não beta-lactâmicos. Assim, de forma geral, o CA-MRSA é susceptível à maioria dos antimicrobianos não beta-lactâmicos. ${ }^{9}$ Essa característica genotípica é expressa na grande maioria em um antibiograma que apresenta resistência apenas ao disco de oxacilina ou cefoxitina, marcadores da resistência aos beta-lactâmicos (Figura 1).

Outra importante característica genética associada com as cepas de CA-MRSA são os genes da citotoxina Panton-Valentine leucocidina (PVL). A PVL é codificada pelos genes lukF e lukS, e sua presença em isolados de $S$. aureus está associada à necrose tecidual e destruição de leucócitos, por meio da formação de poros na membrana celular. ${ }^{10}$ A presença dessa exotoxina pode ser verificada com a pesquisa de genes específicos por reação em cadeia da polimerase (PCR) (Figura 2). Em um estudo realizado nos Estados Unidos, 98\% dos isolados apresentaram genes codificadores da PVL. ${ }^{11}$ As infecções frequentemente associadas com essas cepas são: furúnculos, foliculites, impetigo, artrite séptica, fasciíte necrotizante, pneumonia e septicemia grave. ${ }^{12,13,14}$

Os antimicrobianos orais que são geralmente ativos para as infecções estafilocócicas por CA-MRSA incluem tetraciclinas, trimetropim/sulfametoxazol, fluorquinolonas e clindamicina. Para as formas clíni- 


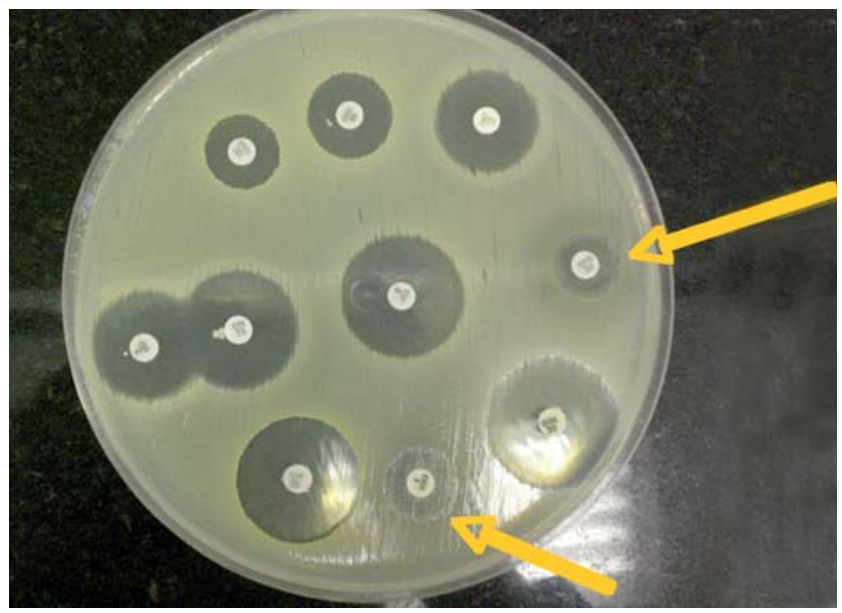

Figura 1: Perfil de susceptibilidade aos antimicrobianos de um isolado de CA-MRSA. As setas indicam os discos de cefoxitina e oxacilina, marcadores da resistência às cefalosporinas e a todos os outros beta-lactâmicos

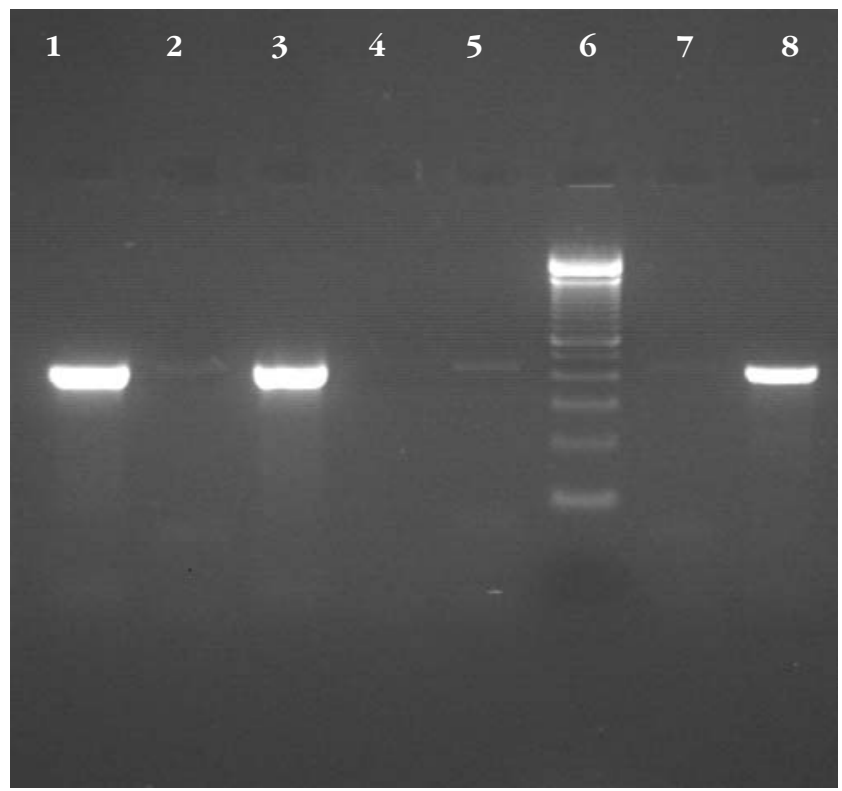

Figura 2: Detecção do gene lukF, codificador da PVL, por PCR Canaleta 1: controle positivo; canaleta 2: controle negativo canaleta 6: marcador de peso molecular (lambda ladder); canaletas 3 e 8: isolados de MRSA positivos para PVL; canaletas 4, 5 e 7: isolados de MRSA negativos para PVL

cas potencialmente graves, relacionam-se: linezolida, tigeciclina, daptomicina, teicoplamina e vancomicina. A clindamicina é um antimicrobiano frequentemente utilizado como estratégia terapêutica no tratamento de infecções estafilocócicas em pele e tecidos por apresentar uma boa penetração e potencial antitoxina. No entanto, a grande preocupação desse tipo de terapia empírica está na expressão da resistência induzível à clindamicina, que pode apresentar no antibiograma resistência à eritromicina e falsa sensibilidade à clindamicina. Esse fenótipo de resistência pode ser identificado pelo laboratório de microbiologia utilizando o teste de susceptibilidade com os discos de eritromicina e clindamicina, o D-teste (disk test). ${ }^{15,16}$

A emergência de isolados de CA-MRSA é considerada preocupante, visto que o tratamento empírico de uma variedade de infecções comunitárias de pele e tecidos moles e até mesmo pneumonias é realizado com antimicrobianos beta-lactâmicos. ${ }^{17}$

Um estudo realizado nos Estados Unidos da América (EUA) mostrou que 80\% dos pacientes com infecções de pele e partes moles receberam tratamento empírico; destes, $57 \%$ não responderam ao tratamento, pois o microrganismo apresentou resistência. Esses achados sugerem a necessidade de reconsiderar as escolhas de tratamentos empíricos para infecções em pele e tecidos moles em áreas onde MRSA é prevalente na comunidade. ${ }^{11}$ Em um hospital-escola da Califórnia - EUA, um estudo demonstrou a presença de $87 \%$ de MRSA entre os $S$. aureus isolados de pacientes apresentando infecções dermatológicas atendidos no setor de emergência. Esse estudo defende a drenagem dos abscessos cutâneos como tratamento preferencial, em contrapartida ao uso de antimicrobianos. $^{18}$

Há algumas controvérsias na escolha do tratamento e até mesmo do antimicrobiano para o manejo das infecções de pele causadas por $S$. aureus. As cefalosporinas estão entre aqueles com maiores índices de utilização.

\section{ASPECTOS CLÍNICOS DA INFECÇÃO POR CA-MRSA}

O primeiro relato de uma forma de CA-MRSA ocorreu em 1993 na Austrália, detectado em populações indígenas locais, ${ }^{19}$ e em 2002 ganhou atenção nos Estados Unidos após surtos de infecções cutâneas em atletas de Los Angeles. ${ }^{20}$ Em 2003, no Missouri - EUA, um estudo realizado entre jogadores profissionais de futebol americano demonstrou a presença de MRSA em lesões de pele em cinco de 58 jogadores do time. ${ }^{21}$

$\mathrm{Na}$ América do Sul infecções causadas por MRSA em pacientes não hospitalizados foram observadas inicialmente em 2001, no Uruguai. A princípio casos esporádicos foram relatados, mas com o subsequente aumento do número de infecções de pele em pacientes da comunidade por esse patógeno houve a caracterização de um surto. ${ }^{22}$ No Brasil, os primeiros isolados caracterizados como CA-MRSA tinham apresentação clínica de furunculose e artrite séptica. ${ }^{17}$

Um estudo multicêntrico realizado na Argentina demonstrou uma alta prevalência de Stapbylococus aureus resistente à meticilina isolado de infecções em crianças provenientes da comunidade. Dentre as 447 amostras analisadas nos anos de 2006 e 2007, 281 se encaixavam nos critérios defini- 
dos para CA-MRSA. Essa investigação demonstrou que $62 \%$ dos isolados eram oriundos de infecções de pele e partes moles. O estudo enfatiza uma revisão urgente na terapia antimicrobiana empírica realizada para o tratamento de lesões de pele em crianças oriundas da comunidade. ${ }^{23}$

A elucidação das infecções causadas por cepas de CA-MRSA é de interesse especial aos dermatologistas, já que têm alta probabilidade de apresentar-se como infecção de pele e tecidos moles. Em um estudo comparativo entre CA-MRSA e HA-MRSA, mostrouse que infecções de pele e tecido subcutâneo eram muito mais prevalentes em pacientes com isolados de CA-MRSA (75\%) do que de HA-MRSA (37\%).

Epidemiologicamente, as infecções relacionadas às cepas de CA-MRSA estão habitualmente associadas a crianças, jovens e pessoas saudáveis, especialmente as que vivem aglomeradas ou que têm estrito contato físico umas com as outras. A grande maioria dos relatos na literatura relaciona atletas de esportes coletivos, indivíduos do serviço militar, encarcerados, usuários de drogas endovenosas, desabrigados e crianças de creches, sendo esses os indivíduos com risco aumentado de desenvolver infecções por CAMRSA. A apresentação típica seria de um jovem atleta com abscesso e celulite adjacente e que provavelmente teria fatores contribuintes como contato físico, dano cutâneo e compartilhamento de equipamento contaminado.

Infecções como a fasciíte necrosante são incomuns por $S$. aureus, porém o crescente número de casos provocados por CA-MRSA se torna preocupante. Em um estudo norte-americano de 2005, 14 de 843 pacientes apresentaram-se com fascí́te necrosante durante um período de 15 meses. O principal fator de risco associado a esses casos foi o uso de drogas injetáveis, e todos os isolados de $S$. aureus mostraram genótipo similar e a presença de genes codificadores da toxina PVL. ${ }^{13}$ Ainda não existem estudos de prevalência no Brasil, mas dados de outros países, como os Estados Unidos, mostram que há uma grande variação de frequência relacionada à geografia. Um estudo conduzido em 11 centros de emergência de diferentes cidades dos EUA, durante agosto de 2004, obteve uma taxa de prevalência global de $59 \%$, com variação entre 15 e $74 \%{ }^{11}$

\section{TIPOS DE CLONES DE CA-MRSA CIRCULANTES}

$\mathrm{Na}$ atualidade, a tipagem molecular tem possibilitado obter novos dados, que dão origem à epidemiologia molecular. No caso de $S$. aureus, recentemente, depois de analisar uma grande variedade de cepas que circulam em diferentes regiões do mundo e em diferentes períodos, encontrou-se que as cepas MRSA têm uma estrutura clonal conservada e que con- tam com um número reduzido de clones com capacidade de disseminação global. ${ }^{6}$

Os primeiros isolados de CA-MRSA foram detectados no oeste da Austrália, no final de 1980, causando infecção na população indígena local; então, foram chamados de WA-MRSA (Western Australian MRSA). ${ }^{19}$ Depois disso, duas outras linhagens de CA-MRSA emergiram na Austrália e na Nova Zelândia: o clone "Queensland" e o clone "Oceania Southwest Pacific" (SWP), também denominado de OSPC ("Oceania South Pacific clone"). ${ }^{24,25}$ Os clones USA foram descritos pela primeira vez nos Estados Unidos da América; o clone USA400 foi relatado no centro-oeste dos Estados Unidos em unidades neonatais e infecções puerperais. O USA300 tem sido relatado em muitas regiões dos Estados Unidos, principalmente em jogadores de futebol americano e presidiários. ${ }^{26} \mathrm{Um}$ estudo de Huang e cols. demonstrou que, dos CA-MRSA isolados em infecções de pele e tecidos moles na Califórnia, 87\% eram clones USA300 ${ }^{27}$ Recentemente, reportou-se que aproximadamente 50\% dos MRSA coletados de infecções de pele e tecidos moles nos Estados Unidos pertencem ao clone USA30011 e menos frequentemente ao USA $400 .{ }^{28}$

No Brasil, os primeiros isolados caracterizados como CA-MRSA eram similares ao clone OSPC e eram provenientes de uma única cidade no sul do país (Porto Alegre). ${ }^{17}$ Posteriormente, um estudo demonstrou a presença desse mesmo clone em isolados na região Sudeste (Rio de Janeiro). ${ }^{29}$ Essas duas áreas geográficas estão separadas por mais ou menos 1.500 quilômetros de distância, demonstrando assim a disseminação do clone OSPC em diferentes regiões do país. O estudo demonstrou também a presença de outras linhagens SCCmec IV classicamente comunitárias, o USA300 e o USA400 recuperados de infecções de pele e pneumonia.

A origem das cepas CA-MRSA, todavia, está sujeita a debates. Uma das possibilidades é a descendência silvestre de cepas hospitalares, ocorrida por meio de uma transformação vertical. Entretanto, num estudo em que se compararam cepas hospitalares com cepas da comunidade, não se encontrou uma relação entre as cepas HA-MRSA e CA-MRSA. Outra possibilidade é a de que as cepas comunitárias surgiram como uma consequência de uma transferência vertical dos genes da resistência à meticilina. A transferência horizontal é possibilitada graças ao complexo do gene ccr que se encontra no SCCmec, que codifica recombinases responsáveis por sua mobilidade. Pensa-se que essa transferência ocorre pouco e que as cepas CA-MRSA são consequência de um desses raros eventos de transferência do gene mec, de um doador a um receptor susceptível. Todas essas hipóteses estão, ainda, sob estudos. ${ }^{6}$ 


\section{CONSIDERAÇÕES FINAIS}

As infecções causadas por MRSA deixaram de ser problemas exclusivos associados ao ambiente hospitalar e estão tornando-se um problema emergente na comunidade. Até o momento, os fatores de risco associados às infecções por MRSA comunitário não estão completamente estabelecidos e o influxo de pacientes ambulatoriais nas unidades de saúde pode ter impacto na epidemiologia intra-hospitalar desse patógeno. É possível que os pacientes colonizados ou infectados por MRSA SCCmec IV permaneçam sem as medidas adotadas para controle da disseminação de MRSA, havendo a possibilidade de disseminação intrahospitalar dessa cepa. Por outro lado, pacientes colonizados por MRSA SCCmec IV podem ser submetidos a cirurgias ou procedimentos em que a profilaxia para MRSA deve ser considerada, aumentando o risco de infecção nesses pacientes.

Sugere-se que, em pacientes ambulatoriais nos quais seja isolado MRSA apresentando susceptibilida- de a vários antimicrobianos, deva ser considerada para o diagnóstico uma possível infecção por CAMRSA. Estudos genéticos incluindo tipagem do gene SCCmec, PFGE (pulsed-field gel electrophoresis) e detecção de genes codificadores da PVL e MLST (multilocus sequence typing) podem ser aplicados para uma melhor caracterização dessa bactéria.

A escassez de estudos de prevalência é um fator limitante do conhecimento da epidemiologia local relacionada ao CA-MRSA. Um aspecto importante que pode facilitar o conhecimento da existência dessas cepas circulantes é o cultivo laboratorial de infecções de pele e tecidos moles em locais de atendimento primário, como as unidades de emergência dos hospitais, ou em centros de assistência dermatológica. Assim, com a identificação do microrganismo e a realização do teste de susceptibilidade, poderá ser instituído um tratamento apropriado e direcionadas as medidas de controle para esse patógeno.

\section{REFERÊNCIAS}

1. Altemeier WA, Culbertson WR, Veto M. Prophylactic antibiotic therapy. AMA Arch Surg. 1955;71:2-6.

2. Barraviera B. Estudo clínico das estafilococcias. J Bras Med. 1994;67:160-92.

3. Ferreira MS, Gonçalves EG, Assis VP. Como diagnosticar e tratar infecções estafilocócicas . Rev Bras Med. 1985;42:179-89.

4. Lowy FD. Staphylococcus aureus infections. N Engl J Med. 1998;339:520-32.

5. Chambers HF. Methicillin resistance in Staphylococci: molecular and biochemical basis and clinical implications. Clin Microbiol Rev. 1997;10:781-91.

6. Bustos-Martínez JA, Hamdan-Partida A, Gutiérrez-Cárdenas M. Staphylococcus aureus: la reemergencia de un patógeno en la comunidad. Rev Biomed. 2006; 17:287-305.

7. Salgado CD, Farr BM, Calfee DP. Community-acquired methicillin-resistant Staphylococcus aureus: a meta-analysis of prevalence and risk factors. Clin Infect Dis. 2003;36:131-9.

8. Forbes BA, Sahm DF, Weissfeld AS. Staphylococcus, Micrococcus, and similar organisms. In: Forbes BA, Sahm DF, Weissfeld AS, editors. Bailey \& Scott's Diagnostic Microbiology. St Louis: Mosby; 1998. p.607-18.

9. Naimi TS, LeDell KH, Como-Sabetti K, Borchardt SM, Boxrud DJ, Etienne J, et al. Comparison of communityand health care-associated methicillin-resistant
Staphylococcus aureus infection. JAMA. 2003; 290: 2976-84.

10. Dufour P, Gillet Y, Bes M, Lina G, Vandenesch F, Floret $\mathrm{D}$, et al. Community-acquired methicillin-resistant infections in France: emergence of a single clone that produces Panton-Valentine leukocidin. Clin Infect Dis. 2002;35:819-24.

11. Moran GJ, Krishnadasan A, Gorwitz RJ, Fosheim GE, Mcdougal LK, Carey RB, et al. Methicillin-resistant $S$. aureus infections among patients in the emergency department. N Engl J Med. 2006; 355:666-74.

12. Lina G, Piemont Y, Godail-Gamot F, Bes M, Peter MO, Gauduchon V, et al. Involvement of Panton-Valentine leukocidin-producing Staphylococcus aureus in primary skin infections and pneumonia. Clin Infect Dis. 1999;29:1128-32.

13. Miller LG, Perdreau-Remington F, Rieg G, Mehdi S, Perlroth J, Bayer AS, et al. Necrotizing fasciitis caused by community-associated methicillin-resistant Staphylococcus aureus in Los Angeles. N Engl J Med. 2005;352:1445-53.

14. Gillet Y, Issartel B, Vanhems P, Fournet JC, Lina G, Bes M, et al. Association between Staphylococcus aureus strains carrying gene for Panton-Valentine leukocidin and highly lethal necrotizing pneumonia in young immunocompetent patients. Lancet. 2002;359:753-9.

15. Chambers HF. MRSA: the San Francisco experience. Program and abstracts of the 45th Interscience 
Conference on Antimicrobial Agents and Chemotherapy; December 16-19, 2005; Washington, DC. Abstract 1324.

16. Patel M, Waites KB, Moser SA, Cloud GA, Hoesley CJ. Prevalence of inducible clindamycin resistance among community and hospital-associated Staphylococcus aureus isolates. J Clin Microbiol. 2006;44:2481-4.

17. Ribeiro A, Dias C, Silva-Carvalho MC, Berquo L, Ferreira FA, Santos RN, et al. First report of infection with community-acquired methicillin-resistant Staphylococcus aureus in South America. J Clin Microbiol. 2005;43:1985-88.

18. Rajendran PM, Young D, Maurer T, Chambers $H$, Perdreau-Remington F, Ro P, et al. Randomized, double-blind, placebo-controlled trial of Cephalexin for treatment of uncomplicated skin abscesses in a population at risk for community-acquired methicillinresistant Staphylococcus aureus infection. Antimicrob Agents Chemother. 2007;51:4044-48.

19. Udo EE, Pearman JW, Grubb WB. Genetic analysis of community isolates of methicillin-resistant Staphylococcus aureus in Western Australia. J Hosp Infect. 1993; 25:97-108.

20. Centers of Disease Control and Prevention. Outbreaks of community-associated methicillin-resistant Staphylococcus aureus skin infections - Los Angeles County, California, 2002-3. MMWR Morb Mortal Wkly Rep. 2003;52:88.

21. Kazakova SV, Hageman JC, Matava M, Srinivasan A, Phelan L, Garfinkel B, et al. A clone of methicillin-resistant Staphylococcus aureus among football players. N Engl J Med. 2005; 352:468-475.

22. Ma XX, Galiana A, Pedreira W, Mowszowicz M, Chrisphersen I, Machiavello S, et al.Communityacquired methicillin-resistant Staphylococcus aureus, Uruguay. Emerg Infect Dis. 2005;11:973-976.

23. Paganini H, Della Latta MP, Opet BM, Ezcurra G, Uranga M, Aguirre C, et al. Estudio multicéntrico sobre las infecciones pediátricas por Stapbylococcus aureus meticilino-resistente provenientes de la comunidad en la Argentina. Arc Argen Pediatr. 2008;106:397-403.

24. Nimmo GR, Schooneveldt J, O'Kane G, McCall B, Vickery A. Community acquisition of gentamicin-sensitive methicillin-resistant Staphylococcus aureus in Southeast Queensland, Australia. J Clin Microbiol. 2000;38:3926-31.
25. Munckhof WJ, Schooneveldt J, Coombs GW, Hoare J, Nimmo GR . Emergence of community-acquired methicillin-resistant Staphylococcus aureus (MRSA) infection in Queensland, Australia. Int Infect Dis. 2003; 7:259-67.

26. Bratu S, Landmand D, Gupta J, Irehan M, Panwar M, Quale J. A population-based study examining the emergente of community-associated methicillin-resistant Staphylococcus aureus USA300 in New York City. Ann Clin Microbiol Antimicrob. 2006;5:29.

27. Huang H, Flynn NM, King JH, Monchaud C, Morita M, Cohen SH. Comparisons of community-associated methicillin resistant Staphylococcus aureus (MRSA) and hospital-associated MRSA infections in Sacramento, California. J Clin Microbiol.2006;44:2423-27.

28. Maree CL, Daum RS, Boyle-Vavra S, Matayoshi K, Miller LG. Community-associated methicillin-resistant Staphylococcus aureus isolates causing healthcareassociated infections. Emerg Infect Dis. 2007;13:236-42.

29. Ribeiro A, Coronado AZ, Silva-Carvalho MC, FerreiraCarvalho BT, Dias C, Rozenbaum R, et al. Detection and characterization of international community-acquired infections by methicillin-resistant Staphylococcus aureus clones in Rio de Janeiro and Porto Alegre cities causing both community-and hospital-associated diseases. Diagn Microbiol Infect Dis. 2007;59:339-45.

\author{
ENDEREÇO PARA CORRESPONDÊNCIA / MAILING ADDRESS: \\ Luciane Cristina Gelatti \\ Laboratório de Microbiologia/Universidade \\ Federal de Ciências da Saúde de Porto Alegre \\ (UFCSPA) \\ Rua Sarmento Leite, 245 - Sala 203 \\ 90050170 Porto Alegre RS \\ Tel.:/Fax: 5133039000 \\ E-mail:lucianegelatti@botmail.com
}

Como citar este artigo/How to cite this article: Gelatti LC, Bonamigo RR, Becker AP, d'Azevedo PA. Staphylococcus aureus resistentes à meticilina: disseminação emergente na comunidade. An Bras Dermatol. 2009;84(5):501-6. 